\title{
Direct evidence of secondary necrosis of neutrophils during intense lung inflammation
}

\author{
K. Rydell-Törmänen, L. Uller and J.S. Erjefält
}

\begin{abstract}
Several pulmonary inflammatory conditions are characterised by infiltration of neutrophils. Normally, neutrophils are silently removed by apoptosis, followed by phagocytosis. However, if phagocytosis fails, apoptotic cells undergo secondary necrosis. Recent findings of increased levels of the pan-necrosis marker lactate dehydrogenase in bronchoalveolar lavage from lipopolysaccharide-exposed mice implies potential involvement of secondary necrosis. Using a similar model, this study aimed to identify the source of lactate dehydrogenase and to search for direct histological evidence of secondary necrosis.

Lipopolysaccharide (LPS) was administered to the lungs of BALB/c mice, and bronchoalveolar lavage and tissue samples were collected 4, 12, 24, 36, 48, 60 and $72 \mathrm{~h}$ after administration. LPS induced a patchy neutrophil-rich lung inflammation, where the numbers of terminal deoxynucleotide transferase-mediated dUTP nick-end labelling-positive neutrophils were increased at $12 \mathrm{~h}$ and onwards. Lavage levels of neutrophils and lactate dehydrogenase increased significantly at 4 and $24 \mathrm{~h}$, respectively. Detailed electron microscopic assessment of neutrophil activation and death modes revealed that up to $14 \%$ of the neutrophils were undergoing secondary necrosis, whereas apoptotic or primary necrotic structural cells were rarely found.

In summary, this study provides direct evidence that secondary necrosis of neutrophils is a common process during intense lung inflammation. This implies that neutrophil apoptosis may cause rather than resolve airway inflammation.
\end{abstract}

KEYWORDS: Apoptosis, endotoxin, inflammation, lactate dehydrogenase, neutrophils

$\mathbf{P}$ ulmonary neutrophilia is a characteristic of several inflammatory lung conditions. Experimental pulmonary exposure of lipopolysaccharide (LPS) induces a fast and intense neutrophil response [1]. Under normal physiological conditions, neutrophils are silently and swiftly eliminated through apoptosis, followed by phagocytosis by alveolar macrophages [2]. However, if the phagocytosis system fails, apoptotic cells die through secondary necrosis, a pro-inflammatory event associated with cell membrane disruption and extracellular spreading of cell contents [3]. Recently, MEDAN et al. [4] suggested that secondary necrosis takes place in inflamed lungs. By using a mouse model of LPSinduced lung inflammation, these authors found the peak of apoptotic cells in bronchoalveolar lavage (BAL) to be followed by an increase in activity of the pan-necrosis marker lactate dehydrogenase (LDH), a phenomenon further aggravated after inhibiting macrophage phagocytosis

For editorial comments see page 257. with phosphatidylserine-containing liposomes [4]. While this important finding suggests secondary necrosis to be a significant feature of LPS-induced lung inflammation, the cell type(s) undergoing secondary necrosis has remained largely unknown. The present study sought to identify the source of LDH in BAL fluid (BALF) via a detailed histological analysis of a LPSinduced inflammation. Special focus was given to neutrophil infiltration and clearance, due to the possibility of finding direct evidence for secondary necrosis of neutrophils to be a significant feature of the inflammation. To allow controlled studies of areas with different intensity of inflammation, a patchy neutrophil-rich lung inflammation was induced in mice using a dual-administration regimen of LPS. At seven different time-points, general inflammation as well as the activity and fate of neutrophils were assessed by BAL studies and lung tissue histology, including an electron microscopic analysis. This approach allowed the current authors to follow the fate of cells within patchy areas of intense neutrophil-rich

\section{AFFLLIATIONS}

Dept of Experimental Medical Science, Division of Vascular and Airway Research, Lund University, Lund, Sweden.

CORRESPONDENCE

K. Rydell-Törmänen

Dept of Experimental Medical

Science

Division of Vascular and Airway

Research

BMC D12

S-221 84 Lund

Sweden

Fax: 46462224546

E-mail: Kristina.Rydell-Tormanen@

med.lu.se

Received:

October 312005

Accepted after revision:

February 202006 
inflammation and infiltration (hereafter referred to as inflammatory foci (IF)) at various phases of lung inflammation in detail, and to study the clearance processes following cell death. The data revealed that the extensive number of cells within IF were neutrophils, of which large numbers were undergoing apoptosis and secondary necrosis, suggesting neutrophils to be the primary source of LDH during an intense lung inflammation. Taking the pathogenic potential of necrosis into consideration $[5,6]$, this study suggests neutrophil secondary necrosis to be a potential pathogenic mechanism during an intense neutrophil-rich lung inflammation.

\section{MATERIAL AND METHODS Mice}

Female BALB/c mice, 6-8 weeks old, were obtained from MoB A/S (Ry, Denmark). Animals were housed in a 12-h light-dark cycle and provided with food and water ad libitum. All protocols were approved by the local ethics committee (Malmö/Lund, Sweden).

\section{Lipopolysaccharide-induced lung inflammation}

A total dose of $50 \mu \mathrm{g}$ LPS (Escherichia coli (O26:B6); Sigma, St Louis, MO, USA) was administered intra-nasally during a light anaesthesia, using a dual-administration regimen. This administration of LPS has, in preliminary experiments, been shown to produce a patchy lung inflammation with areas of variable inflammatory intensity and neutrophil infiltration. Animals were sacrificed $4,12,24,36,48,60$ and $72 \mathrm{~h}$ after LPS administration $(n=5$ in each group; controls $(n=5)$ received double administration of saline) by pentobarbital sodium (Pentobarbitalnatrium; Apoteket AB, Umeå, Sweden). BALs were performed on all animals as previously described [7] and tissue samples were obtained for paraffin (via immunohistochemistry) and plastic embedding (transmission electron microscopy (TEM)) [7]. The rationale for analysing BAL and tissue samples from the same animals was to investigate the total contents of the lung, both what could be retrieved by BAL and what was left in the lung after BAL.

\section{Bronchoalveolar lavage fluid}

The BALF was centrifuged, the supernatant immediately frozen on dry ice and the cell pellet resuspended in PBS supplemented with $10 \%$ foetal calf serum. The BALF level of the cytoplasm enzyme LDH was used as a general marker of cell membrane disruption, i.e. necrosis. The activity of LDH in BAL was enzymatically determined (LDH converted lactate and nicotinamide adenine dinucleotide to pyruvate and reduced nicotinamide adenine dinucleotide, resulting in increased absorbance that was directly proportional to the activity of $\mathrm{LDH}$; the analysis was performed using a Hitachi Modular-P unit; Roche Diagnostics, Basel, Switzerland) in $100 \mu$ l of BALF, by the Laboratory of Clinical Chemistry (Lund University Hospital, Lund, Sweden). Differential cell counting was preformed on May-Grünewald/Giemsa-stained cytospin slides.

\section{Histological assessment of inflammation}

Histological observations, such as extent of inflammation, infiltrating cells and occurrence of cell debris, were examined on 6- $\mu \mathrm{m}$ haemotoxylin and eosin-stained paraffin sections, costained with the DNA-marker Hoechst 33342 (H33342; Sigma, St Louis, MO, USA). Neutrophils were identified as cells with polymorph nucleus, apoptotic cells by intensely DNA-stained condensed nuclei, and necrotic cells and cell debris as vague and blurry positive DNA staining. The extent of inflammation was determined as the degree of cellular infiltrate in lung parenchyma, classified into three categories: 1) no or mild cellular infiltration; 2) extensive infiltration with partly filled alveoli; and 3) extensive cellular infiltrate with filled alveolar spaces. Categories 2 and 3 were defined as IF.

\section{Detection of apoptosis}

The terminal deoxynucleotide transferase-meditated dUTP nick-end labelling (TUNEL) technique, performed according to the manufacturer's protocol (Intergen Company, New York, NY, USA) as previously described [8], in combination with H33342, was used to identify apoptotic cells in lung sections, as discussed in another study [9]. When using the TUNEL technique it is important to consider that not only apoptotic cells are labelled, but also necrotic cells and free apoptotic cell nuclei stain positive. Therefore, no separation of these stages was performed using this analysis; the differentiation was performed with electron microscopy. For quantification, highresolution digital images of the lung parenchyma were randomly obtained (4-6 images per slide) and the total number of TUNEL-positive cells/nuclei in each image was counted (blinded) using the Image $J_{\circledR}$ software $(1.30 \mathrm{v}$; National Institutes of Health, Bethesda, MD, USA) and expressed as cells $\cdot \mathrm{mm}^{-2}$. For quantification of positivity within areas of intense neutrophil infiltration and inflammation (IF), selected areas within sections displaying numerous TUNEL-positive staining were photographed and analysed as previously described.

\section{Transmission electron microscopy}

TEM analysis was performed to achieve detailed structural analysis. Cells in different modes of activation (i.e. neutrophils with granule alterations and/or phagosomes) and death were quantified using set and previously validated criteria (table 1; fig. 1) [10-13]. At least 90 neutrophils per time-point were counted, with the exception being controls for which a very limited number of neutrophils were found, and the results were expressed as percentage of the total number of cells. The areas studied via TEM were consciously chosen to include IF, as the aim of the present study was to investigate the inflammatory process in these areas.

\section{Data analysis and statistics}

Analyses were made as previously described. The KruskallWallis test was used to assess differences; when significant, the Mann-Whitney test was employed to explore the differences between groups. All groups were compared to control. Unless otherwise stated, data were given as median (range) and $p \leqslant 0.05$ was considered statistically significant.

\section{RESULTS}

\section{Characterisation of inflammation}

The number of neutrophils in BALF increased significantly $4 \mathrm{~h}$ after LPS administration, peaked at $36 \mathrm{~h}$ and returned towards baseline levels at $72 \mathrm{~h}$ (table 2). The level of the necrosis marker LDH in BALF increased significantly in response to LPS (table 2). The level of LDH peaked at $60 \mathrm{~h}$ and correlated with the number of neutrophils in BAL. In the tissue, a 


\begin{tabular}{ll} 
TABLE 1 & Criteria used for electron microscopy quantification of activity status following lipopolysaccharide administration \\
\multicolumn{1}{c}{ Status } & Characterisation features \\
\hline Resting & Intact cells with normal nucleus, intact cell membrane and normal primary and secondary granules \\
Activated & One or more of the following features: granule alterations, vacuoles, phagosomes, extensive pseudopodia \\
Apoptosis & Intact cell membrane, condensed and electron dense nucleus, often with blebs along the nuclear membrane \\
$\begin{array}{l}\text { Primary necrosis } \\
\text { Secondary necrosis }\end{array}$ & Ruptured cell membrane and normal or chromatolytic nucleus \\
Cell debris & As apoptosis but with a ruptured cell membrane \\
\hline
\end{tabular}
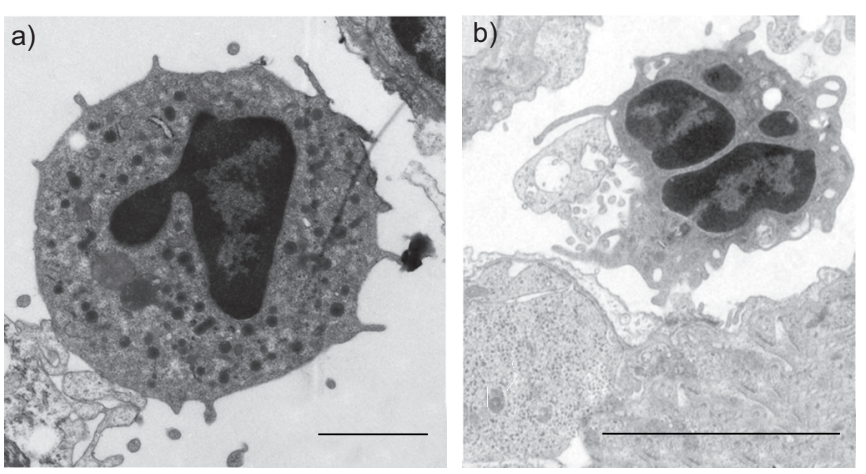

c)

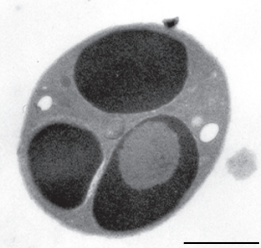

d)
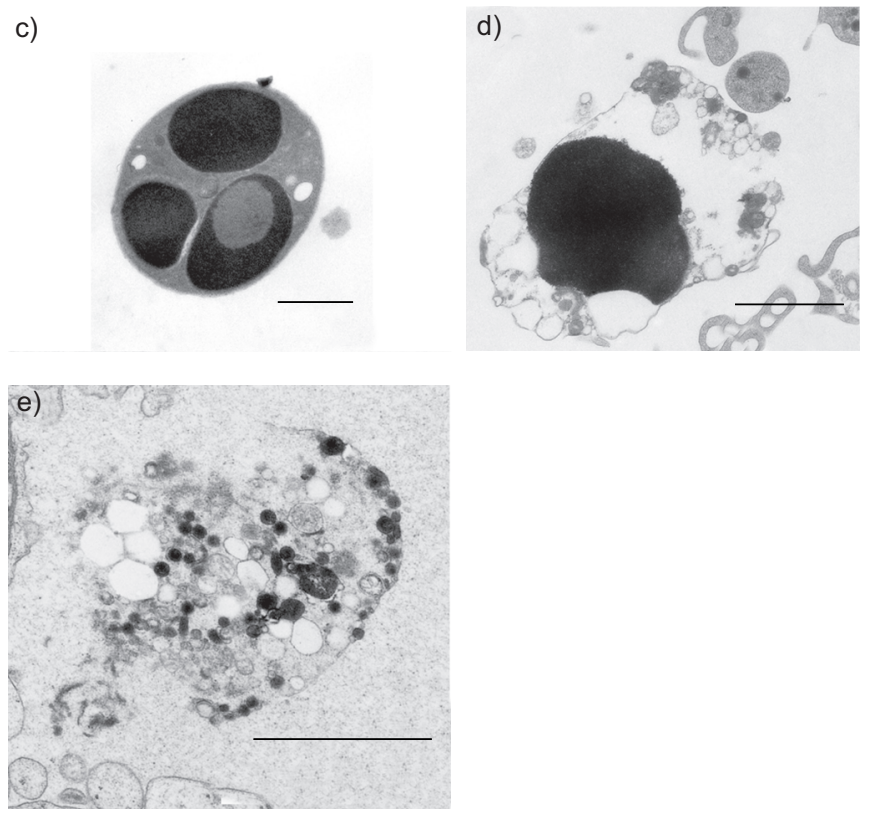

patchy neutrophil-rich inflammatory pattern was confirmed by histological analysis of the lung parenchyma. Thus, cell-dense IF were present amidst lung regions with a mild-to-moderate neutrophilia (fig. 2a and b). IF (extent of inflammation categories 2 and 3) were characterised by extensive cellular infiltrate that completely or partly filled the alveolar spaces and by plugs in the luminal spaces of bronchi and bronchioles. The most common cell type in IF was neutrophils, followed by macrophages. Neutrophils were also the most abundant cell type in cell-rich plugs found in the luminal spaces of bronchi and bronchioles in IF (fig. 2c).

\section{Focal accumulation of TUNEL positivity}

Whereas the total number of TUNEL-positive cells increased dramatically after LPS administration, the augmentation was most prominent in IF (table 2). Based on cell-specific morphological criteria the TUNEL-positive cells were identified as being primarily neutrophils, but also scattered macrophages and structural cells stained positively.

\section{Cellular fate in IF}

The most abundant cell type in IF was the neutrophil, but macrophages were also present in relatively large numbers. Generally, in IF, neutrophils inside the alveolar wall or the subepithelial tissue surrounding bronchi and bronchioles were at rest showing little or no activation. Scattered neutrophils in these tissue compartments did undergo apoptosis, but no apoptotic neutrophils were found inside pulmonary capillaries. In contrast, resting neutrophils were rarely observed in the alveolar lumen; the vast majority of the neutrophils were activated with granulae alterations and/or intracellular phagosomes (fig. 3a). A marked increase in apoptosis, and primary and secondary necrosis as well as cell debris (i.e. extracellular neutrophil granules, free condensed nuclei and other cell remnants) was regularly seen in IF $24 \mathrm{~h}$ after LPS administration (fig. $3 a$ and $b$ ). The proportion of different activation statuses and death modes of alveolar neutrophils varied between time-points (fig. 4). From $4 \mathrm{~h}$ after LPS administration, the majority of the neutrophils were displaying an activated phenotype. At later time-points, the number of apoptotic neutrophils increased, as did the number of neutrophils undergoing secondary necrosis. Neutrophils undergoing primary necrosis were found, though only a few scattered cells, indicating that the increase in neutrophilderived cell debris was mainly due to secondary necrosis. The TEM analysis further revealed that scattered structural cells (i.e. type I and II pneumocytes) exhibited signs of cell damage, such as a pale and swollen cytoplasm and cell membrane 


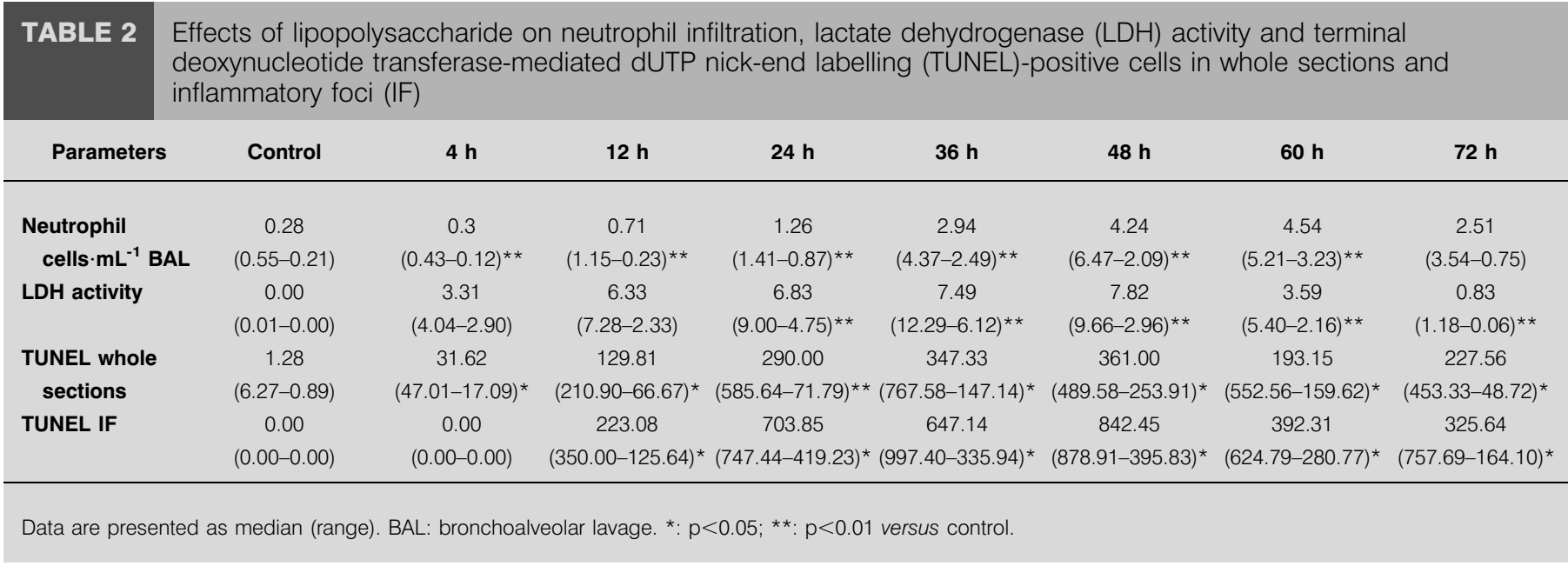

rupture indicating an ongoing necrosis process (fig. 3c); fewer displayed an apoptotic phenotype. The vast majority of the alveolar macrophages in IF contained multiple large phagosomes, and occasional macrophages displayed signs of necrosis, revealed by chromatolytic nucleus- and electronlucent cytoplasm.
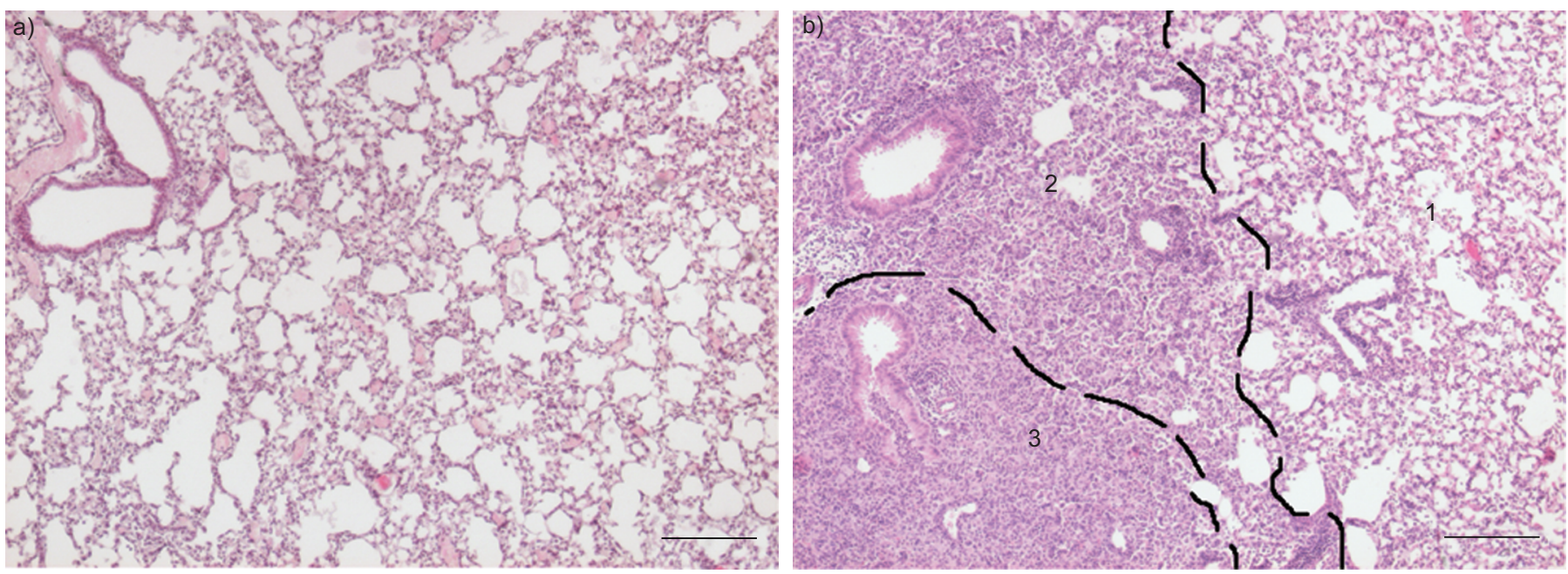

\section{DISCUSSION}

There is abundant evidence supporting a critical role for apoptosis and subsequent phagocytosis in swift and silent clearance of peripheral airway neutrophils [2]. This study investigated the clearance of neutrophils during LPS-induced lung inflammation and sought to identify the source of the

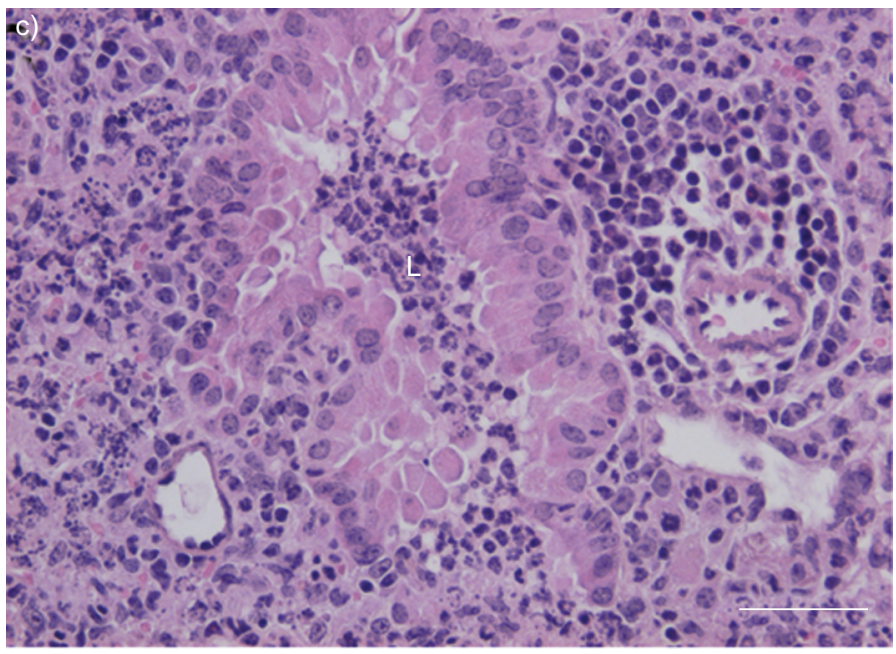

FIGURE 2. Representative bright-field microscopic illustrations of haemotoxylin and eosin-stained sections from a) a control animal exposed to saline and b) an animal $60 \mathrm{~h}$ after lipopolysaccharide (LPS) administration. An inflammatory focus, characterised by an extensive cellular infiltration filling the alveolar spaces (3) is easily separated from adjacent regions of more moderate infiltration with partly filled alveolar spaces (2) and normal lung parenchyma (1). Inflammatory foci were defined as categories 2 and 3. c) In areas of intense inflammation (60 h after LPS administration), not only were the alveolar spaces filled with cell infiltrates but the lumen of bronchi and bronchioles (L) were frequently occluded. Scale bars $=100 \mu \mathrm{m}$ ( $\mathrm{a}$ and b) and $50 \mu \mathrm{m}$ (c). 

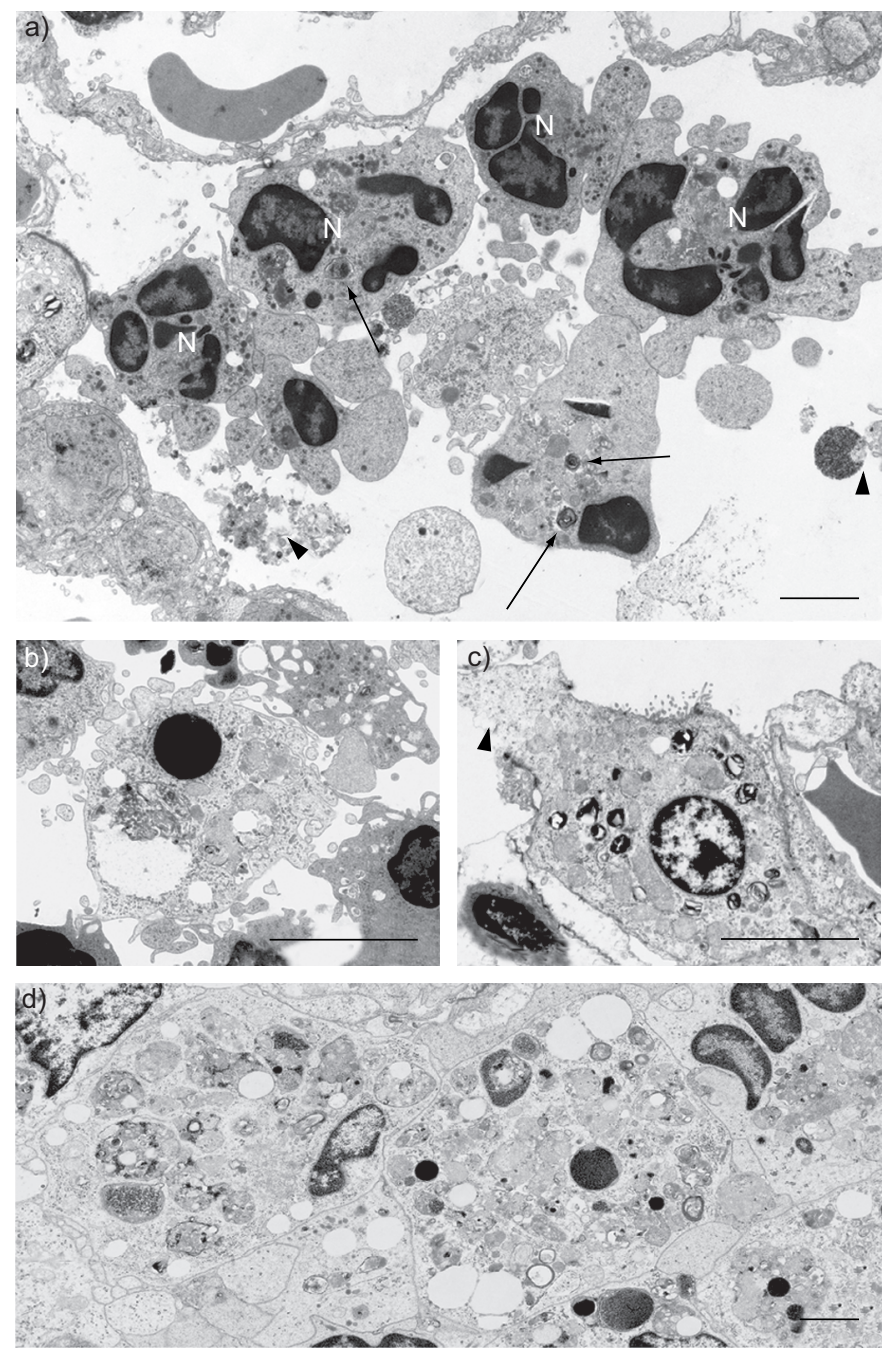

FIGURE 3. Transmission electron micrographs demonstrating characteristic features of neutrophils, structural cells and macrophages in inflammatory foci (IF). a) Neutrophils (N) were primarily activated with granulae alterations or phagosomes (indicated by arrows) in lumen amidst cell debris (arrowheads), such as free apoptotic nuclei and neutrophil-derived granulae. b) Secondary necrosis of neutrophils (cells with condensed and electron-dense nuclei and ruptured cell membrane) were extensively seen in IF. c) Only scattered dying structural cells were found, illustrated by a type-Il pneumocyte undergoing primary necrosis, with early signs of chromatolysis and rupture of the cell membrane (arrowhead). d) In IF, macrophages commonly contained multiple phagosomes. Scale bars $=2.5 \mu \mathrm{m}(\mathrm{a})$, $5 \mu \mathrm{m}(\mathrm{b}$ and $\mathrm{c}$ ) and $2 \mu \mathrm{m}(\mathrm{d})$.

necrosis marker LDH, previously suggested to increase follwing endotoxin exposure [4]. The present study found that apoptotic neutrophils in IF are not properly cleared and therefore undergo secondary necrosis in large numbers.

In light of the pro-inflammatory potential of secondary necrosis $[4,6,14]$, surprisingly little is known about its occurrence and role in inflamed lungs. A relationship between apoptosis and LDH levels has been shown in BAL samples of LPS-treated mice, a phenomenon further increased following interference with macrophage phagocytosis by phophatidylserine-containing liposomes [4]. However, as LDH is a pan-necrosis marker it may be released by any cell

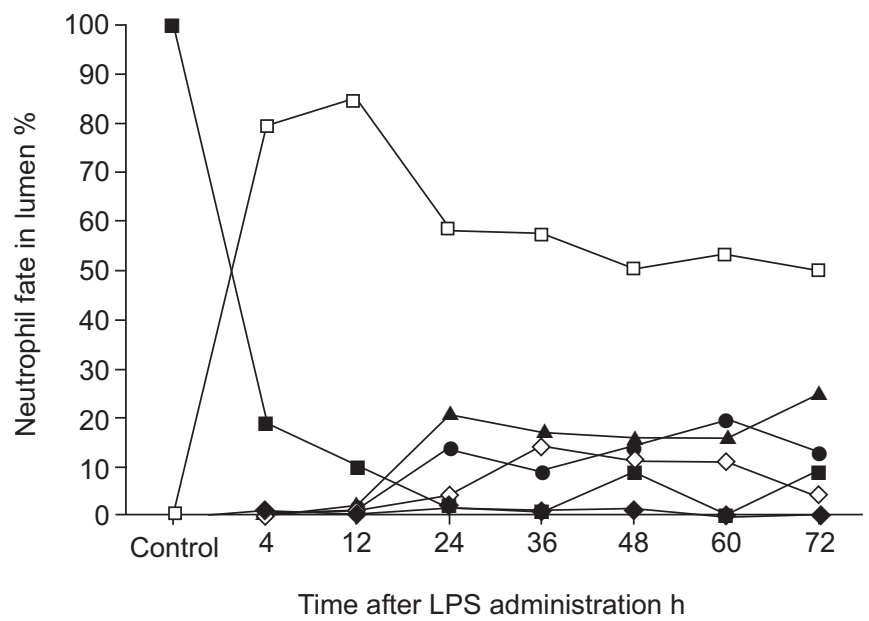

FIGURE 4. A detailed transmission electron microscopy analysis was performed to reveal the fate of neutrophils in cell-rich inflammatory foci at different time-points following lipopolysaccharide (LPS) administration. The fates of the neutrophils were divided into six categories depending on the following morphological characteristics: resting ( $\mathbf{\square}$; intact cells, normal nucleus and granulae), activated ( $\square$; granulae alterations, vacuoles, phagosomes and/or extensive pseudopodia), apoptosis ( $\bullet$; intact cell membrane, condensed nucleus, often blebs along the nuclear membrane), primary necrosis ( $\bullet$; ruptured cell membrane and normal/chromatolytic nucleus), secondary necrosis $(\diamond$; as apoptosis but ruptured cell membrane) and cell debris ( $\mathbf{\Lambda}$; extracellular gatherings of neutrophil-derived material). The data are given as percentages of different categories of total number of neutrophils at each time-point.

undergoing either primary or secondary necrosis. Several fundamental questions therefore remain regarding the nature of secondary necrosis in the lung. For example, it is still unclear which cell types actually undergo secondary necrosis, and are therefore the source of the LDH found in BAL, and if/how this release relates to modes of cell death, such as primary necrosis [13]. Furthermore, until now, virtually nothing has been known about when and where in the inflammatory process secondary necrosis takes place.

This study shows that in IF, only scattered pneumocytes displayed ultrastructural signs of apoptosis, whereas signs of primary necrosis (electron-lucid cytoplasm and damaged cell membrane, for example) were more commonly found. Although this finding supports the notion that necrosis rather than apoptosis is the usual death mode of structural cells [15], it must not be forgotten that apoptosis of structural cells occurs more frequently in more chronic situations. The study also found numerous apoptotic neutrophils and neutrophils in the process of secondary necrosis, suggesting that the apoptotic process is not too swift to be detected. This implies that apoptotic neutrophils undergoing secondary necrosis are the primary source of LDH, and the fact that secondary necrotic neutrophils occurred amidst apoptotic neutrophils and extracellular neutrophil-derived cell debris suggests a rapid turnover of neutrophils.

In general, the present data support the notion of apoptosis and phagocytosis as a highly effective neutrophil-elimination mechanism $[3,16]$. As previously described, LPS induced a patchy inflammation [1], with regions of no or mild 
inflammation amidst areas of extensive inflammation. In the regions of mild and moderate inflammation, apoptosis and subsequent macrophage phagocytosis carried out swift and silent neutrophil elimination, resulting in sufficient clearance of infiltrating neutrophils. A similar silent removal process may also have occurred in IF, to an as yet unknown extent. However, as demonstrated through TEM analysis, in IF a significant proportion of the neutrophils underwent secondary necrosis, suggesting the clearance to be insufficient. Taken together, the present data suggest secondary necrosis to be a rare phenomenon in large areas of the lung parenchyma (with mild-to-moderate neutrophil infiltration) but a common fate of lung neutrophils in IF.

Little is known about secondary necrosis in relevant clinical situations. It is clear, however, that secondary necrosis occurs extensively in the airway lumen of patients with chronic obstructive pulmonary disease (COPD), as assessed by TEM analysis of directly fixated sputum samples [17]. Hence, secondary necrosis seems to be a common fate of senescent and apoptotic cells trapped in airway mucus plugs. To what extent such luminal necrosis affects the underlying airway mucosa is currently unknown, although it has recently been demonstrated in vitro that neutrophils undergoing secondary necrosis have the capacity to damage airway epithelial cells [6], suggesting a potentially pathogenic role of luminal secondary necrosis. Human data on secondary necrosis in the more fragile peripheral airways are lacking. From the present study, it can be concluded that the most likely site for secondary necrosis to occur is in areas of intense inflammation and neutrophil infiltration, IF. As demonstrated by histopathological and lung-imaging techniques, areas with a degree of inflammation similar to the IF examined in the present model occur frequently during common lung infections [18, 19], for example, and probably during COPD exacerbations. This indicates that similar processes to the one currently described in mouse lungs may in fact be part of several clinical conditions.

Interestingly, macrophages exposed to smoke or collected from COPD patients have been shown to exhibit an impaired phagocytic capacity $[20,21]$; similar results have been obtained with LPS-stimulated alveolar macrophages from patients suffering from severe asthma [22]. It can therefore be speculated that the extensive secondary necrosis found in the present model is due to impairments in the macrophage clearance system. In support of this, the present study revealed that the vast majority of the macrophages within IF contain multiple large phagosomes and had occasionally started to disintegrate in necrosis. However, to clarify whether the macrophage clearance system is impaired or overwhelmed, further investigations on this subject and the role of secondary necrosis in the pathogenesis of common airway diseases are required.

In summary, this study demonstrates that in acutely inflamed lungs insufficient clearance of apoptotic neutrophils results in secondary necrosis, a pro-inflammatory process in which intracellular components are released. The increases in lactate dehydrogenase seen following lipopolysaccharide exposure originate mainly from secondary necrosis of apoptotic neutrophils, rather than from secondary necrosis of structural cells. The process of secondary necrosis may thus play a role in the pathogenesis of neutrophil-rich inflammatory airway conditions, such as chronic obstructive pulmonary disease exacerbations and infectious pneumonia.

\section{ACKNOWLEDGEMENTS}

The authors would like to thank K. Jansner for invaluable support with animal handling and procedures, and B-M. Nilsson for assistance with sample preparation for electron microscopy.

\section{REFERENCES}

1 Domenici L, Pieri L, Galle MB, Romagnoli P, Adembri C. Evolution of endotoxin-induced lung injury in the rat beyond the acute phase. Pathobiology 2004; 71: 59-69.

2 Cox G, Crossley J, Xing Z. Macrophage engulfment of apoptotic neutrophils contributes to the resolution of acute pulmonary inflammation in vivo. Am J Respir Cell Mol Biol 1995; 12: 232-237.

3 Haslett C. Granulocyte apoptosis and its role in the resolution and control of lung inflammation. Am J Respir Crit Care Med 1999; 160: S5-S11.

4 Medan D, Wang L, Yang X, Dokka S, Castranova V, Rojanasakul Y. Induction of neutrophil apoptosis and secondary necrosis during endotoxin-induced pulmonary inflammation in mice. J Cell Physiol 2002; 191: 320-326.

5 Fadok VA, Bratton DL, Guthrie L, Henson PM. Differential effects of apoptotic versus lysed cells on macrophage production of cytokines: role of proteases. J Immunol 2001; 166: 6847-6854.

6 Liu CY, Liu YH, Lin SM, et al. Apoptotic neutrophils undergoing secondary necrosis induce human lung epithelial cell detachment. J Biomed Sci 2003; 10: 746-756.

7 Korsgren M, Persson CG, Sundler F, et al. Natural killer cells determine development of allergen-induced eosinophilic airway inflammation in mice. J Exp Med 1999; 189: 553-562.

8 Erjefalt JS, Uller L, Malm-Erjefalt M, Persson CG. Rapid and efficient clearance of airway tissue granulocytes through transepithelial migration. Thorax 2004; 59: 136-143.

9 Uller L, Persson CG, Kallstrom L, Erjefalt JS. Lung tissue eosinophils may be cleared through luminal entry rather than apoptosis: effects of steroid treatment. Am J Respir Crit Care Med 2001; 164: 1948-1956.

10 Erjefalt JS, Persson CG. New aspects of degranulation and fates of airway mucosal eosinophils. Am J Respir Crit Care Med 2000; 161: 2074-2085.

11 Majno G, Joris I. Apoptosis, oncosis, and necrosis. An overview of cell death. Am J Pathol 1995; 146: 3-15.

12 Uller L, Andersson M, Greiff L, Persson CG, Erjefalt JS. Occurrence of apoptosis, secondary necrosis, and cytolysis in eosinophilic nasal polyps. Am J Respir Crit Care Med 2004; 170: 742-747.

13 Erjefalt J. Transepithelial migration, necrosis and apoptosis as silent and pro-inflammatory fates of airway granulocytes. Curr Drug Targets Inflamm Allergy 2005; 4: 425-431.

14 Hallett MB, Lloyds D. Neutrophil priming: the cellular signals that say "amber" but not "green". Immunol Today 1995; 16: 264-268.

15 Wickenden JA, Clarke MC, Rossi AG, et al. Cigarette smoke prevents apoptosis through inhibition of caspase activation 
and induces necrosis. Am J Respir Cell Mol Biol 2003; 29: 562-570.

16 Henson PM, Bratton DL, Fadok VA. Apoptotic cell removal. Curr Biol 2001; 11: R795-R805.

17 Erjefalt JS, Larsson S, Persson C, Nihlen U, Lofdahl CG, Greiff L. Necrosis of neutrophils and eosinophils together with granulocyte-derived granule proteins in directly fixed sputum samples from COPD patients. Am J Respir Crit Care Med 2002; 165: A598.

18 Hammerschmidt DE. Lobar pneumonia. J Lab Clin Med 2004; 143: 327.

19 Hacimustafaoglu M, Celebi S, Sarimehmet H, Gurpinar A, Ercan I. Necrotizing pneumonia in children. Acta Paediatr 2004; 93: 1172-1177.
20 Hodge S, Hodge G, Scicchitano R, Reynolds PN, Holmes M. Alveolar macrophages from subjects with chronic obstructive pulmonary disease are deficient in their ability to phagocytose apoptotic airway epithelial cells. Immunol Cell Biol 2003; 81: 289-296.

21 Prieto A, Reyes E, Bernstein ED, et al. Defective natural killer and phagocytic activities in chronic obstructive pulmonary disease are restored by glycophosphopeptical (inmunoferon). Am J Respir Crit Care Med 2001; 163: 1578-1583.

22 Huynh ML, Malcolm KC, Kotaru C, et al. Defective apoptotic cell phagocytosis attenuates prostaglandin e2 and 15-hydroxyeicosatetraenoic acid in severe asthma alveolar macrophages. Am J Respir Crit Care Med 2005; 172: 972-979. 